\title{
The LHCb VErtex LOcator
}

\author{
D. Eckstein on behalf of the LHCb Collaboration ${ }^{1}$
}

\begin{abstract}
The dedicated $\mathrm{CP}$ violation experiment at the $\mathrm{LHC}$, LHCb, will be equipped with a novel silicon detector (VELO). The VELO will provide precise measurements of tracks from displaced $b$-vertices and will allow to trigger on them. The entire detector will be housed in a mobile secondary vacuum system, and after the injection and stabilisation of the beams each fill, the silicon detectors will move inwards and approach to within $7 \mathrm{~mm}$ of the beams. In order to fulfil the trigger requirements, the VELO must combine in an unprecedented way the use of high resolution silicon detectors and large CPU farms. The extreme, non-uniform radiation environment puts additional constraints on the sensor design. The design of the VELO is described, along with the R\&D of the silicon sensors and its production status.
\end{abstract}

\section{Introduction}

At the Large Hadron Collider a high yield of about $10^{12} b \bar{b}$-pairs per year will be produced in $14 \mathrm{TeV} p p$-collisions. The LHCb experiment is dedicated to the precise determination of $\mathrm{CP}$ violating observables making use of this large $b$-hadron statistics. Measurements of $\mathrm{CP}$ violation using many different $b$-hadron decay modes will over-constrain the prediction of the Standard Model and will reveal possible inconsistencies pointing to New Physics.

The LHCb detector is designed as a single arm spectrometer to exploit the strongly correlated, forward peaked production of $b \bar{b}$-hadrons. It covers an angular range of $15-$ $300 \mathrm{mrad}$. The tasks of the Vertex Locator (VELO) in LHCb are to reconstruct the production and decay vertices of $b$-hadrons, to reconstruct tracks covering the full angular acceptance of the downstream detectors and to provide information for the second level trigger.

For a precise vertexing it is important to have short extrapolation distances to the vertex and minimal multiple scattering between the first measured point and the vertex. These requirements lead to a layout as shown in Fig. 1 with 21 silicon stations placed perpendicular to the beam over a distance of $1 \mathrm{~m}$ around the interaction region covering completely the $\mathrm{LHCb}$ acceptance and partly the upstream hemisphere for a better primary vertex determination. As a good signal-to-noise performance for at least 2 years is required while the detector is operating in a high and non-uniform radiation environment, special attention was given to the sensor and front-end chip R\&D.

\footnotetext{
${ }^{1}$ Proceedings of a talk presented at the $9^{\text {th }}$ Pisa Meeting on Advanced Detectors, La Biodola, Isola d'Elba, Italy, May 25-31, 2003
} 


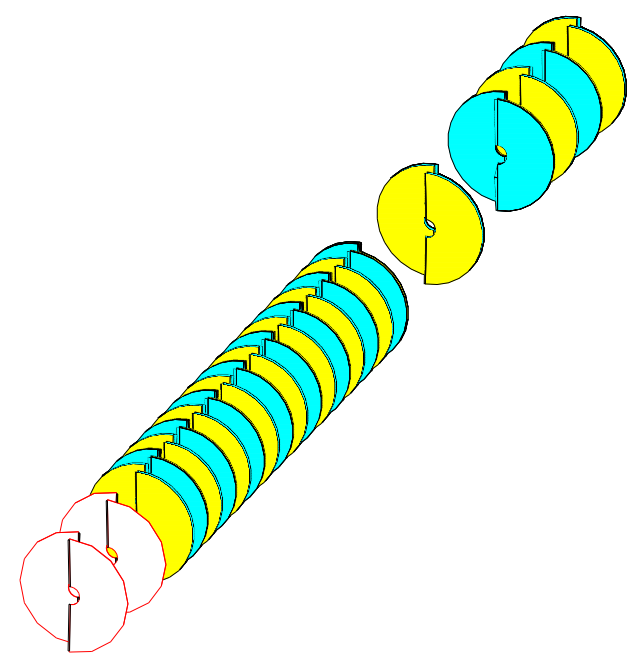

Figure 1: Schematic of the VELO sensor arrangement.

\section{Mechanical Design of the VELO}

To allow measurements as close as possible to the interaction vertex the VELO sensors approach the beam by $7 \mathrm{~mm}$, a distance smaller than the aperture required during injection. Thus the sensors are placed in two independent detector boxes which can be retracted by $30 \mathrm{~mm}$ from the nominal position. In order to cover the full azimuthal acceptance and for aligning the left and the right detector halves with respect to each other, the modules are displaced by $1.5 \mathrm{~cm}$ along the beam line and the sensors overlap.

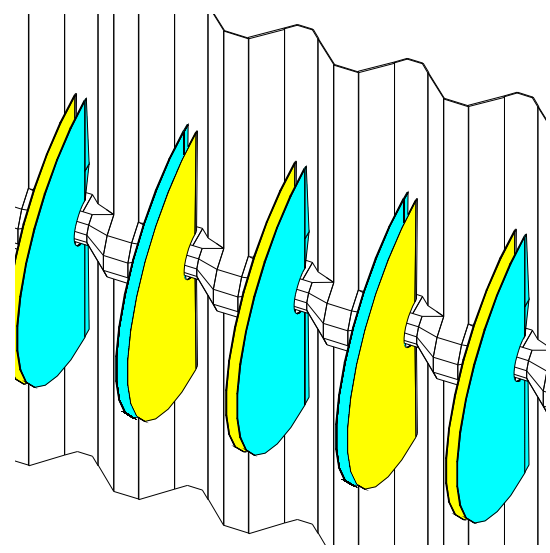

Figure 2: Schematic of the RF-foil together with silicon sensors.

For protecting the LHC vacuum from out-gassing of the VELO modules and for shielding the sensors against RF pickup, the sensors are mounted in a thin aluminium box under vacuum. The box also acts as a wake-field guide for the LHC beams and needs therefore to be connected to the LHC beam pipe via flexible joints. The part of the box facing the LHC beam, RF-foil, has a complicated structure as shown in Fig. 2. Deep corrugations around the beam axis reduce the amount of material traversed by a particle before its first 
measurement in the silicon sensors to a minimum. The outer corrugations are formed to allow the overlap of the two offset detector halves. As material for the foil an aluminium alloy with 3\% magnesium was chosen, AlMg3. Prototype full-size RF-foils were produced using a hot gas forming method and tested for vacuum tightness and mechanical rigidity. The minimal thickness so far was $300 \mu \mathrm{m}$, prototypes with $250 \mu \mathrm{m}$ and $200 \mu \mathrm{m}$ foils will follow.

More details about the mechanical design can be found in [1]. Changes made to the VELO design in the context of the recent overall optimisation of the LHCb experiment can be found in $[2]$.

\section{Sensor Design}

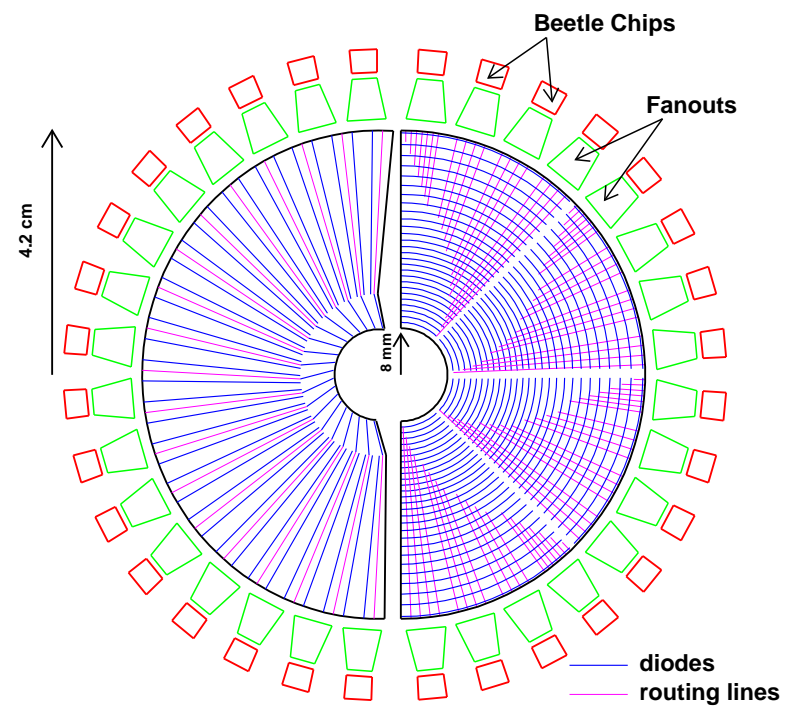

Figure 3: Schematic of the VELO sensor design, left: $\phi$-measuring sensor, right: Rmeasuring sensor.

The design of the VELO sensors is shown in Fig. 3. One reason for the chosen R$\phi$-geometry was to optimise the stand-alone tracking performance for the second level trigger. The two types of AC-coupled, single-sided sensors will be mounted back-to-back within one station.

The strips of the $\phi$-measuring sensors are quasi-radial and segmented in an inner and an outer region in order to equalise the occupancy. The signal from the inner region is routed to the read-out chips located in the outer part of the sensor via lines in a second metal layer. The strips are tilted with a stereo angle different in sign and magnitude for the inner and outer regions and the detectors are flipped from station to station.

The R-sensor strips are of circular shape, thus providing a measurement of the Rcoordinate of hits. Also here the signal from the strips is routed to the detector edge via routing lines in a second metal layer. In the design as described in the TDR [1], the strips in the R-sensor are segmented into 4 azimuthal sections at inner radii $<24.1 \mathrm{~mm}$ and into 2 sections at radii above. 


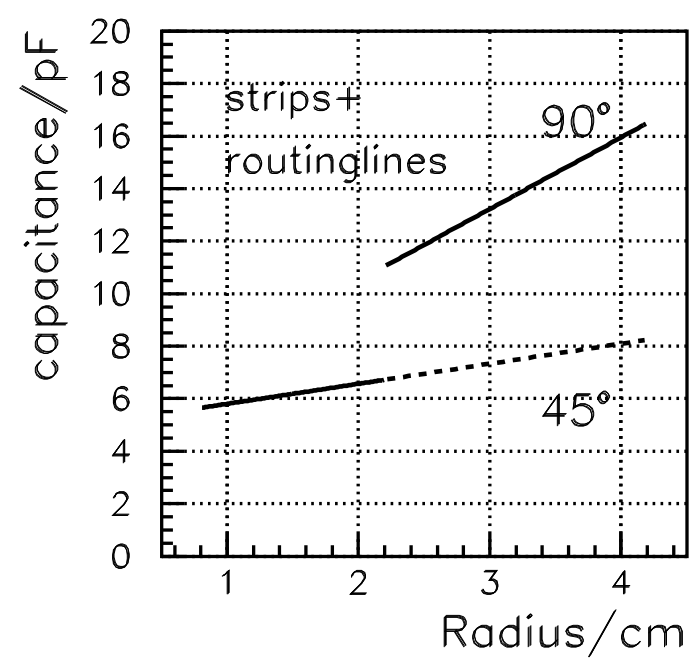

Figure 4: Capacitance as a function of radius introduced by the strips and the routinglines for the old design (solid lines) and the new design (labelled $45^{\circ}$ ).

Investigations of the VELO tracking performance in the context of the optimisation of the LHCb design showed, that the number of clone and ghost tracks as well as the execution time of the track reconstruction can be improved by subdividing the strips into 4 sections at all radii, see [3]. Another benefit is the improved uniformity of the capacitance and its reduction in the outer sector as shown in Fig. 4. Therefore also the noise will be more regular for the changed design.

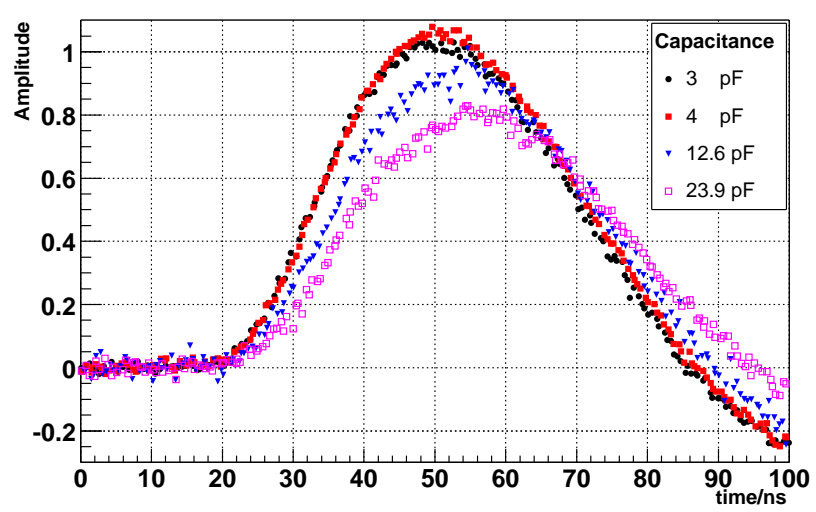

Figure 5: Pulse shapes of the Beetle1.2 front-end chip for different load capacitances.

The rise-time of a pulse of the front-end chip as well as the remainder after $25 \mathrm{~ns}$ (the time, the next event might be triggered), depend on the load capacitance, as shown in Fig. 5 for the Beetle1.2 front-end chip. Only one sampling time can be used for 16 chips reading out a whole sensor. Thus the choice of the optimal sampling time, where the signal is maximal and the remainder minimal, will benefit from a uniform capacitance in the sensor and the signal and remainder variation will be small. 
In order to keep the number of channels at 2048, the strips in the new layout with 4 sectors had to be redistributed. Three different designs were studied and the effect on the impact parameter resolution was compared to the TDR design. It was shown, that the loss in impact parameter resolution is small and reaches at maximum $5 \%$.

\section{Radiation environment}

Due to the position of the VELO very close to the beam, the detectors have to operate in an extreme radiation environment. Moreover, the irradiation will be strongly non-uniform and will vary from $1.3 \times 10^{14} \mathrm{n}_{e q} / \mathrm{cm}^{2} /$ year at smallest radii in the sensor closest to the interaction, to $5 \times 10^{12} \mathrm{n}_{e q} / \mathrm{cm}^{2} /$ year at the largest radii of the sensor furthest away. The effects of irradiation were studied in great detail with different prototype sensors. The results lead to the choice of n-on-n sensors for the VELO and are described in [1].

\section{Front-End Chip}

There were two parallel developments followed for front-end chips satisfying the LHCb requirements. The development of the SCTA_VELO [4] started from the SCT128A chip developed for ATLAS, which is built in the DMILL process. The Beetle chip is built in $0.25 \mu \mathrm{m}$ CMOS technology and is a successor of the HELIX chip developed for the HERA-B experiment.

An extensive test program was in place in order to prepare the decision for one of these chips due in January 2003. The performance of both, the SCTA_VELO and the by then available version of the Beetle chip, the Beetle1.1, was investigated in the test-beam in summer 2002. The main goal was to prove, that the chips were able to read out a whole VELO sensor of a design close to the final with the required signal-to-noise performance. For this purpose hybrids for each chip type were designed, built and equipped with 16 chips reading out a full VELO prototype p-on-n R-measuring sensor.

First results of the measurement with the SCTA_VELO chips are described in [5]. A signal-to-noise of $\approx 16$ was measured. The reported results for the Beetle1.1 chips [6] was a signal-to-noise of $\approx 18$ for a spill-over (remainder after $25 \mathrm{~ns}$ ) of $36 \%$. Thus both chips fulfil the requirements of the LHCb VELO. For mainly the reasons of availability of the CMOS technology also in the future, and the usage of the Beetle chip also in other detectors of the $\mathrm{LHCb}$ experiment [7], the Beetle was chosen as front-end chip for the VELO.

Currently, an improved version, the Beetle1.2, is being tested in laboratory and testbeam measurements. For an efficient operation of the VELO also in the trigger a high signal-to-noise should be accompanied by a small spill-over in order to have a small number of clusters left over from a previous event. A spill-over of $36 \%$ as measured for the Beetle1.1 is too high, but this value is expected to be smaller for the Beetle1.2. In addition, the Beetle has several parameters in order to tune the pulse shape. These influence the signal amplitude, the rise-time and the spill-over. Most important parameters are the shaper current, the pre-amplifier current and the feedback-resistance of the shaper.

As for the measurements with the Beetle1.1, the settings of the Beetle1.2 were scanned to find the optimal working point. Moreover, the 1.3 version of the Beetle, with a few 
final optimisations, will be delivered in autumn and tested with a radioactive source in the laboratory.

\section{Conclusions}

The design of the LHCb VELO has undergone important optimisations and is now close to completion. Production of the Vacuum Vessel has started. A first complete silicon module is expected for the end of this year. The decision was taken to use the Beetle front-end chip for reading out the VELO sensors. More tests will be performed with the current version of the Beetle in order to improve the performance.

\section{References}

[1] The LHCb Collaboration, LHCb Vertex Locator Technical Design Report, CERN/LHCC/2001-011 (2001).

[2] The LHCb Collaboration, Status of the LHCb Detector Reoptimisation, CERN/LHCC/2003-003 (2003).

[3] L. Wiggers et al., R-Sensor sectors and Strip Pitch, LHCb 2003-012, VELO (2003).

[4] J. Buytaert, SCTA_VELO, LHCb 2001-045, VELO (2001).

[5] J. Buytaert et al., A Measurement of the SCTA_VELO signal-to-noise performance on a full-size hybrid, LHCb-2003-033, VELO (2003).

[6] S. Klous et al., Characteristics of 16 Beetle1.1 chips on a VELO hybrid, LHCb-note in preparation, LHCb-2003-069.

[7] D. Baumeister, Beetle - A Radiation Hard Readout Chip for the LHCb-Experiment, these proceedings. 